\title{
Cardiac Electrophysiology Studies Based on Image and Machine Learning
}

\author{
Yashbir Singh ${ }^{1}$, Deepa ${ }^{1}$, Shi-Yi Wu ${ }^{1}$, Michael Friebe², João Manuel R. S. Tavares ${ }^{3}$, Weichih Hu, ${ }^{1, *}$ \\ ${ }^{1}$ Department of Biomedical Engineering, Chung Yuan Christian University, Zhongli, Taiwan \\ ${ }^{2}$ Electrical Engineering and Information Technologies, Otto-von-Guericke-University, Magdeburg, Germany \\ ${ }^{3}$ Instituto de Ciência e Inovação em Engenharia Mecânicae Engenharia Industrial, Departamento de Engenharia Mecânica, Faculdade \\ de Engenharia, Universidade do Porto, Porto, PORTUGAL \\ *Corresponding author: weichihhu@cycu.edu.tw
}

\begin{abstract}
Cardiac Electrophysiology study is the origin and treatment of arrhythmia, which is an abnormality in the rate, regularity or sequence of cardiac activation. There has been considerable recent development in this field, where computational methods such as Imaging and Machine Learning for Cardiac Electrophysiology, provide the framework for cardiac re-modeling. In this research, we review various recent strategies currently available for the meeting the goal of structurally and functionally integrated models of cardiac function that combine data intensive cellular systems models with compute-intensive anatomically detailed multiscale simulations.
\end{abstract}

Keywords: cardiac electrophysiology, cardiac re-modelling, cardiac imaging, machine learning

Cite This Article: Yashbir Singh, Deepa, Shi-Yi Wu, Michael Friebe, João Manuel R. S. Tavares, and Weichih Hu, "Cardiac Electrophysiology Studies Based on Image and Machine Learning." Journal of Biomedical Engineering and Technology, vol. 6, no. 1 (2018): 1-6. doi: 10.12691/jbet-6-1-1.

\section{Introduction}

The human cardiovascular system is the naturally specialized system which circulates oxygenated blood to the other body parts and collects deoxygenated blood at the same time. The non-stop function of this system is the key to our existence. However, a major number of surprise deaths occur due to malfunction associated with the cardiac system and nowadays, electrophysiology related malfunctions are high in the count. Arrhythmia is one of the serious issues associated with the electrophysiology in which heartbeat deviates from its usual behavior and abnormal heartbeat occurs. It is seen that arrhythmia occurs by the formation of the combination of pro arrhythmic substrate, triggers, and modulators. To curb this problem, prior precaution and diagnosis are necessary, yet the human cardiac electrical system is not a plain-sailing task. Medication can be helpful at a certain stage but for a better cure, there should where we can do more. The issue is quite challenging and complex, however, Electrophysiologists are working hard to deal with the more complex organ constituents e.g. the left atrium and complex, often repaired, congenital heart disease, as well as more complex circuits, e.g. atypical atrial flutters, often after prior surgery or ablation procedures [1]. Electro physiologically related problems as a concern in imaging patients, heart rate, and ectopy are important factors [2]. A number of Imaging techniques are offering aid in diagnosing cardio electrophysiology pathologies. Apart from the other cardiac problems, electrophysiology issues must be corrected during live sessions of the cardiac anatomy. Most imaging techniques are majorly reliable on either invasive methods or exposure of radiations to depict the problem. A lot of other imaging techniques like standard fluoroscopy, computed tomography (CT) and magnetic resonance imaging (MRI) are helpful. There are also many other imaging modalities obtained during a procedure, such as those obtained utilizing catheter movement - the so-called non-fluoroscopic mapping systems, those obtained using some form of ultrasound, such as transesophageal (TEE) and intracardiac (ICE) echocardiography, in either 2D or 3D form, and various forms of cardiac MRI, either intracardiac catheter-based MRI, or utilizing experimental MRI EP laboratories [1]. The non-invasive imaging techniques like Standard Electrocardiogram (ECG), Epicardial activation map, Integral maps and Non-invasive imaging of cardiac electro physiology (NICE) [3]. But to develop cost-effective real-time assessment techniques to diagnose the cardio electrophysiology pathologies can assist in disseminating the error-free information of the affected area of the patient for the further treatment procedure. Modern cardiac electrophysiology studies include catheter-based arrhythmia ablation and transvenous device implantation, which are highly dependent on accurate, real-time cardiac imaging and machine learning [4]. This era has been led to an explosion of advance technology especially machine learning. Rapidly increasing in biological data dimension and acquisition rate is demanding conventional analysis strategies [5]. Modern machine learning methods are like deep learning and convolutional neural network, promise to influence very large data sets for finding hidden arrangement within them, and for making more precise predictions. Present 
time, cardiac Electrophysiologists are handling more complex procedures that involve very complex anatomy. The goals of the newer techniques are varying to decrease radiation exposure to patient and physician, to improve the efficacy of the procedures, their outcomes, safety, and to allow Electrophysiologists to tackle more complex procedures [6,7]. In medical science, we usually prefer medical image dataset for a Machine learning application which is adequate data volume, annotation, and reusability. Each cardiac image data consist data elements and an identifier. Such combination exhibits an imaging examination. The dataset must have enough imaging examinations to answer the question being asked. To maximize algorithm development, both the dataset itself and each imaging examination must be described and labelled accurately $[8,9]$. Here in this paper, we tried to describe the already present techniques of imaging and what can be possible in the future in respect of machine learning with the cardiac image.

\section{Materials and Methods}

We reported the criteria used to identify the relevant works for the review performed in this domain. We followed the PRISMA statement for the design, implementation, analysis, and reporting of the results [10].

\subsection{Selection Method}

A search was performed on Pub Med (827 Articles), Scopus (197 Articles), and ISI Web of Sciences databases (24 Articles) 2000 to 2017. The search used the exact expression "Cardiac electrophysiology." Only works published between 2000 and 2017 were considered. A total of distinct articles were obtained and evaluated and followed the same method using the keyword "Machine Learning on cardiac image". We got 128 articles on Pub Med, 6 articles on Scopus and 55 articles on ISI Web of Sciences databases.

\subsection{Data Collection and Processing}

The identification of the relevant works was performed through double-stage strategy, as explained below and previously reported in PRISMA guidelines. First, the title and the abstract of all articles obtained, analyzed by the first author of the current study [10]. Second, the obtained 225 articles were completely read and analyzed, and the following criteria were used to identify the relevant studies: (1) the selected studies should focus on novel technical innovations of the cardiac electrophysiology, discarding small case-to-case adaptations of the traditional approach due to difficult intervention or strange anatomies; (2) About the current cardiac electrophysiology technique were also considered as valid for this review; (3) Short clinical reports, letters between editor, author or reader and short communications without technical description were discarded; (4) previously presented reviews about cardiac electro physiology and similar topics were also rejected. As such, a total of 123 articles were selected for the review. Note that the identification of the relevant works was done by the first author of this review [10].

\subsection{Data Analysis}

The 123 selected articles were fully analyzed and classified into different categories: incidence studies, preprocedural planning methods, surgical instruments and machine learning approach. Each publication could be included in more than one category [10].

\section{Results}

\subsection{Study Characteristics}

Most reports suggest that machine learning and cardiac imaging has become effective technique at this time. A lot of applications of machine learning have been proposed for feature extraction and segmentation of cardiac images. This technique can be utilized for automatic detection of lesions on coronary computed tomography angiography images. Improvements for automatic lesion localization have been demonstrated by support vector machine method, which integrated many quantitative geometric and shape features (including stenosis, minimum luminal diameter, circularity, eccentricity), resulting in high sensitivity, specificity, and accuracy (93\%, 95\%, 94\%). Very recently, machine learning techniques have been applied for the identification of calcified plaques on computed tomography angiography images, demonstrating improved accuracy over the existing methods (Table 1).

\subsection{Incidence Study}

Many researches contain heterogeneous populations that involve abnormal atria anatomy. The research shows that approximately $12 \%$ of the procedures require more than one complications/failures rate. It may be noted that since studies with different operator's expertise (i.e., expert and trainee), heterogeneous populations, and multiple surgical types of equipment were included in this analysis, high variation of complication and failure rate was found.

\subsection{Recent cardiac Imaging Techniques}

There are few cardiac imaging techniques are involved in cardiac electrophysiology which is the most effective way to discover and analyze the problem. A number of imaging techniques are available: Rotational angiography: This imaging technique is used to obtain 3D CT-like images using X-rays with a high-speed rotation of C-arm. It provides clear, accurate and high-quality images in preparation for surgery. This technique can be applied for pre-surgical imaging of various vascular anomalies [21]. Pre-operative CT or MRI: These two imaging techniques are quite similar in their processing part. They both provide the cross-sectional images of the body organs. However, the imaging techniques are non-identical. CT scanners use X-rays across the body part to obtain many cross-sectional view images while MRI scanners use strong magnetic fields and radio frequencies to obtain the detailed images of the organ. The obtained results help in diagnosis and what should be the next step to deal with the problem. 
Table 1. Summary of Machine Learning Algorithms Applied in Cardiovascular Imaging Studies

\begin{tabular}{|c|c|c|c|c|}
\hline $\begin{array}{c}\text { Author ' s } \\
\text { Article (Years) }\end{array}$ & Study Design & Techniques & Measures & Major Findings \\
\hline $\begin{array}{l}\text { Berchialla et al } \\
\text { [11] (2012) }\end{array}$ & $\begin{array}{l}\text { Cross-sectional } \\
\text { study }\end{array}$ & $\begin{array}{l}\text { Bayesian network/ Logistic } \\
\text { Regression/Random forest/ } \\
\text { Artificial Neural Network/ } \\
\text { Support Vector Machine }\end{array}$ & $\begin{array}{l}\text { Used data computed tomography } \\
\text { angiography to predict future } \\
\text { cardiovascular events }\end{array}$ & $\begin{array}{l}\text { Measurement of Left Ventricle } \\
\text { dysfunction }\end{array}$ \\
\hline $\begin{array}{l}\text { Isgum et al [12] } \\
\qquad(2012)\end{array}$ & $\begin{array}{l}\text { Longitudinal } \\
\text { study }\end{array}$ & $\begin{array}{l}\text { k- nearest neighbor/ Support } \\
\text { Vector Machine }\end{array}$ & $\begin{array}{l}\text { Score of coronary calcium in } \\
\text { low-dose, noncontrast enhanced } \\
\text { chest computed tomography } \\
\text { scans }\end{array}$ & $\begin{array}{l}\text { Classification with k- nearest } \\
\text { neighbor with selected features) } \\
\text { Detected on average (sensitivity } \\
79.2 \% \text { ) of coronary Ca volume } \\
\text { with average } 4 \text { mm false-positive } \\
\text { volume }\end{array}$ \\
\hline $\begin{array}{l}\text { Lee et al [13] } \\
\quad(2013)\end{array}$ & $\begin{array}{l}\text { Cross-sectional } \\
\text { study }\end{array}$ & $\begin{array}{l}\text { Decision tree//k- nearest } \\
\text { neighbor /Support Vector } \\
\text { Machine }\end{array}$ & $\begin{array}{l}\text { Analyze abdominal aortic } \\
\text { aneurysm geometry on contrast } \\
\text { CT images and wall surface } \\
\text { curvatures predict rupture risk }\end{array}$ & $\begin{array}{l}\text { k- nearest neighbor demonstrated } \\
\text { the more accuracy } 85.5 \% \text {, an } \\
\text { accuracy of Support Vector } \\
\text { Machine, decision tree, and naive } \\
\text { Bayes was } 83.4 \% \text {, } 83.3 \% \text {, and } \\
80.1 \% \text {, respectively }\end{array}$ \\
\hline $\begin{array}{l}\text { Mohammadpour } \\
\text { et al [14] (2015) }\end{array}$ & $\begin{array}{c}\text { Cross-sectional } \\
\text { study }\end{array}$ & $\begin{array}{l}\text { Fuzzy rule-based classifying } \\
\text { system }\end{array}$ & $\begin{array}{l}\text { Myocardial perfusion scan and } \\
\text { clinical variables to predict } \\
\text { coronary artery disease }\end{array}$ & $\begin{array}{l}\text { Determined important risk factors } \\
\text { for coronary artery disease }\end{array}$ \\
\hline $\begin{array}{l}\text { Xiong et al [15] } \\
\text { (2015) }\end{array}$ & $\begin{array}{l}\text { Cross-sectional } \\
\text { study }\end{array}$ & Naive Bayes/Random forest & $\begin{array}{l}\text { Determine physiologically a } \\
\text { manifestation of coronary } \\
\text { stenosis by assessing myocardial } \\
\text { perfusion on computed } \\
\text { tomography angiography images }\end{array}$ & $\begin{array}{l}\text { Diagnosis of obstructive coronary } \\
\text { artery stenosis. Accuracy } 0.70 \text {, } \\
\text { Sensitivity } 0.79 \text {, and Specificity } \\
0.64\end{array}$ \\
\hline $\begin{array}{l}\text { Knackstedt et al } \\
\text { [16] (2015) }\end{array}$ & $\begin{array}{l}\text { Cross-sectional } \\
\text { study }\end{array}$ & $\begin{array}{l}\text { Vendor independent software } \\
\text { Auto LV }\end{array}$ & $\begin{array}{l}\text { Measure Left ventricle volumes, } \\
\text { Ejection Fraction, and average } \\
\text { biplane longitudinal strain using } \\
\text { ultrasound images }\end{array}$ & $\begin{array}{l}\text { Left Ventricle Ejection Fraction } \\
\text { and longitudinal strain assessment }\end{array}$ \\
\hline $\begin{array}{l}\text { Arsanjani et al } \\
\text { [17] (2015) }\end{array}$ & $\begin{array}{l}\text { Longitudinal } \\
\text { study }\end{array}$ & $\begin{array}{l}\text { Machine learning an Logit } \\
\text { Boost }\end{array}$ & $\begin{array}{l}\text { Single positron emission } \\
\text { computed tomography perfusion } \\
\text { data to predict early } \\
\text { revascularization in patients with } \\
\text { suspected coronary artery } \\
\text { disease; }\end{array}$ & $\begin{array}{l}\text { Logit Boost predict } \\
\text { revascularization, total ischemic } \\
\text { perfusion of the heart }\end{array}$ \\
\hline $\begin{array}{l}\text { Berkel et al [18] } \\
\quad \text { (2016) }\end{array}$ & $\begin{array}{l}\text { Longitudinal } \\
\text { study }\end{array}$ & $\begin{array}{l}\text { Artificial neural network } \\
\text { /Naive Bayes/ Logistic } \\
\text { regression }\end{array}$ & $\begin{array}{l}\text { Diagnose acute coronary } \\
\text { syndrome and electro } \\
\text { echocardiography findings }\end{array}$ & $\begin{array}{l}\text { Accuracy of artificial neural } \\
\text { network, naive Bayes, logistic } \\
\text { regression found } 91.26 \%, 88.75 \% \text {, } \\
\text { and } 90.1 \%\end{array}$ \\
\hline $\begin{array}{l}\text { Celutkiene et al } \\
\text { [19] (2016) }\end{array}$ & $\begin{array}{l}\text { Longitudinal } \\
\text { study }\end{array}$ & $\begin{array}{l}\text { Custom multiparametric } \\
\text { mathematical model }\end{array}$ & Detection of myocardial ischemia & $\begin{array}{l}\text { Myocardial ischemia in patients } \\
\text { with coronary stenosis } \geq 50 \% \text { with } \\
\text { sensitivity } 91.6 \% \text { and specificity } \\
86.3 \% \text {, compared with } 76.8 \% \text { and } \\
89 \% \text {. }\end{array}$ \\
\hline $\begin{array}{l}\text { Motwani et al } \\
\text { [20] (2017) }\end{array}$ & $\begin{array}{l}\text { Longitudinal } \\
\text { study }\end{array}$ & $\begin{array}{l}\text { Custom-built predictive } \\
\text { classifier }\end{array}$ & $\begin{array}{l}\text { Predict 5-year all-cause mortality } \\
\text { in patients with suspected } \\
\text { coronary artery disease } \\
\text { undergoing coronary computed } \\
\text { tomography angiography }\end{array}$ & $\begin{array}{l}\text { Area Under Curve was } 0.79 \text { vs } 0.61 \\
\text { for Framingham risk score, } 0.64 \text { for } \\
\text { segment stenosis score, } 0.64 \text { for } \\
\text { segment involvement score }\end{array}$ \\
\hline
\end{tabular}

Non-fluoroscopic mapping systems: A catheter introduced into the system to model 3D structures of heart chambers. The application of this non-fluoroscopic catheter visualization system (NFCV) reduces fluoroscopy time and dose by $90-95 \%$ in a variety of electrophysiology (EP) procedures. This can be of relevance not only to the patients but also to the nurses and physicians working in the EP lab [22]. Image fusion with non-fluoroscopic mapping systems: The images are fused by using anatomic information obtained pre- or intra-operatively to fuse with an anatomic model made with the use of a fluoroscopic mapping system so as to obtain a better representation of the true anatomy of a chamber [23]. Echocardiographic imaging: An echocardiogram is used to obtain the images of heart by using two-dimensional, three-dimensional and Doppler ultrasound. The 3D images are obtained in real time with Trans thoracic and transesophageal echocardiography and present off-line with intracardiac echocardiography [23]. The real-time and near real-time imaging of chambers and structures are also lesion discoveries [24]. Intracardiac MRI: A catheter is used to analyze the internal structure or any other difficulty in heart, likewise similar to intracardiac echocardiography [23].

\subsection{Machine Learning Techniques}

The first important task is to acquire high-quality imaging data. No matter how flexible, all algorithms will require some interface customization with respect to 
locale, institution, and modality-specific configurations and infrastructures. As cardiovascular imaging modality techniques continue to evolve, algorithms also need to accommodate measures (ie, variables) and layers of imaging data added over time; thus, highly customized algorithms may need substantial edits to handle new data types (Figure 1). With respect to optimizing image quality, filtering and other algorithms can be applied in real time even though inter-individual variation in clinical characteristics will always lead to variations in image quality. Traditionally, data analysis involves trained technicians selecting anatomic structures and performing measurements that are over-read by a cardiologist or radiologist who often adds diagnostic information to the record. The data are then combined with clinical data into a single dataset, and conventional statistics are used to determine whether a given measurement is relevant to a clinical outcome. Here I have mentioned few feature which helps in the study of cardiac electrophysiology study. Velocity features, Circumferential strain, Radial strain, Local and global Simpson Volume and Segmental Volume of the cardiac image are the features where we get information to study electrophysiology of the heart.

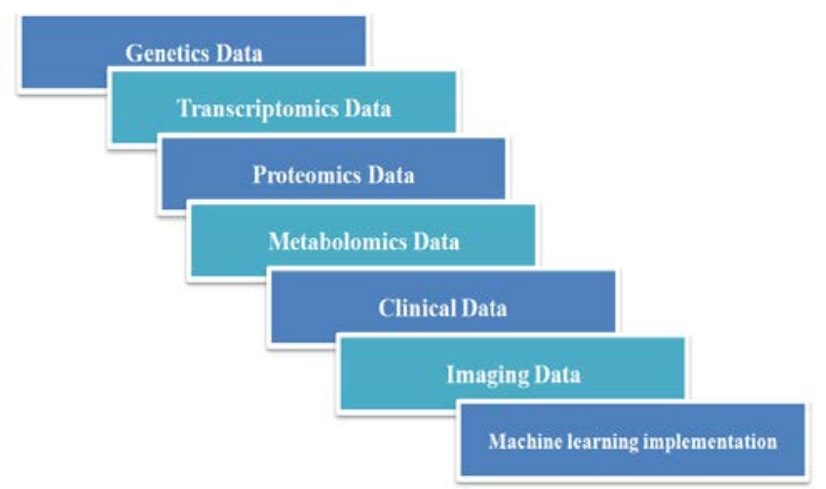

Figure 1. Machine learning implementation on different kind of data

There are few recent techniques of machine learning which is mentioned basically.

\subsubsection{Deep Learning}

This has become one of the most dynamic fields in machine learning that mimics the cognitive processing of the human brain using neural networks with multiple hidden layers. Deep architectures trained on millions of photographs can famously detect objects in pictures better than humans do. All current models in image classification, image retrieval and semantic segmentation make use of neural networks. At a pixel level, the convolution operation scans the image with a given pattern and calculates the strength of the match for every position $[25,26]$. There are also various medical imaging modalities obtained during a procedure, such as those obtained utilizing catheter movement the so-called nonfluoroscopic mapping systems, those obtained using some form of ultrasound, such as transesophageal (TEE) and intracardiac (ICE) echocardiography, in either 2D or 3D form, and various forms of cardiac MRI, either intracardiac catheter-based MRI, or utilizing experimental MRI EP laboratories [27,28,29,30]. Pooling confirms the existence of the pattern in a region, for example by calculating the maximum pattern match in smaller patches (max-pooling), thereby aggregating region information into a single number. The successive application of convolution and pooling operations is at the core of most network architectures used in image analysis [31].

\subsubsection{Artificial Neural Network}

An artificial neural network consist layers of interconnected compute units. The depth of a neural network corresponds to the number of hidden layers and the width to the maximum number of neurons in one of its layers. As it became potential to train networks with numerous hidden layers, artificial neural networks were rebranded to "deep networks". In the canonical configuration, the network receives data in an input layer, which are then transformed in a nonlinear way through multiple hidden layers, before final outputs are computed in the output layer (Figure 2) [32,33].

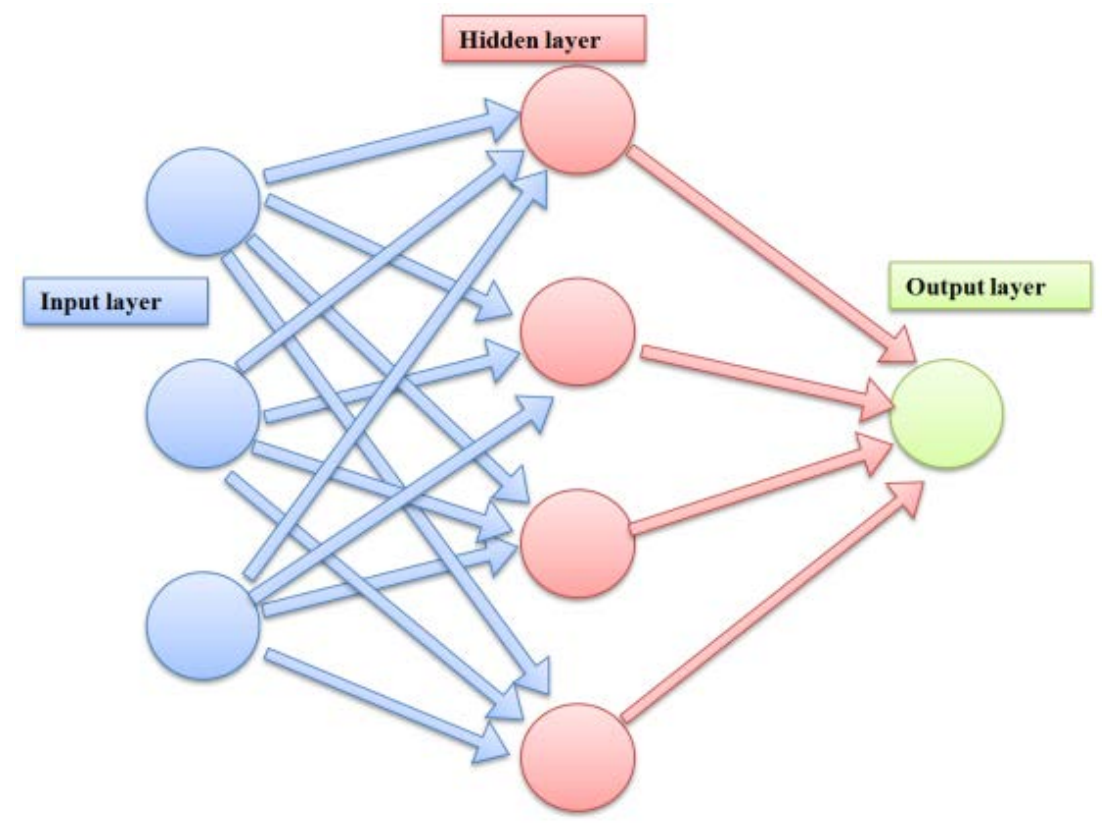

Figure 2. Diagram of Artificial Neural Network 


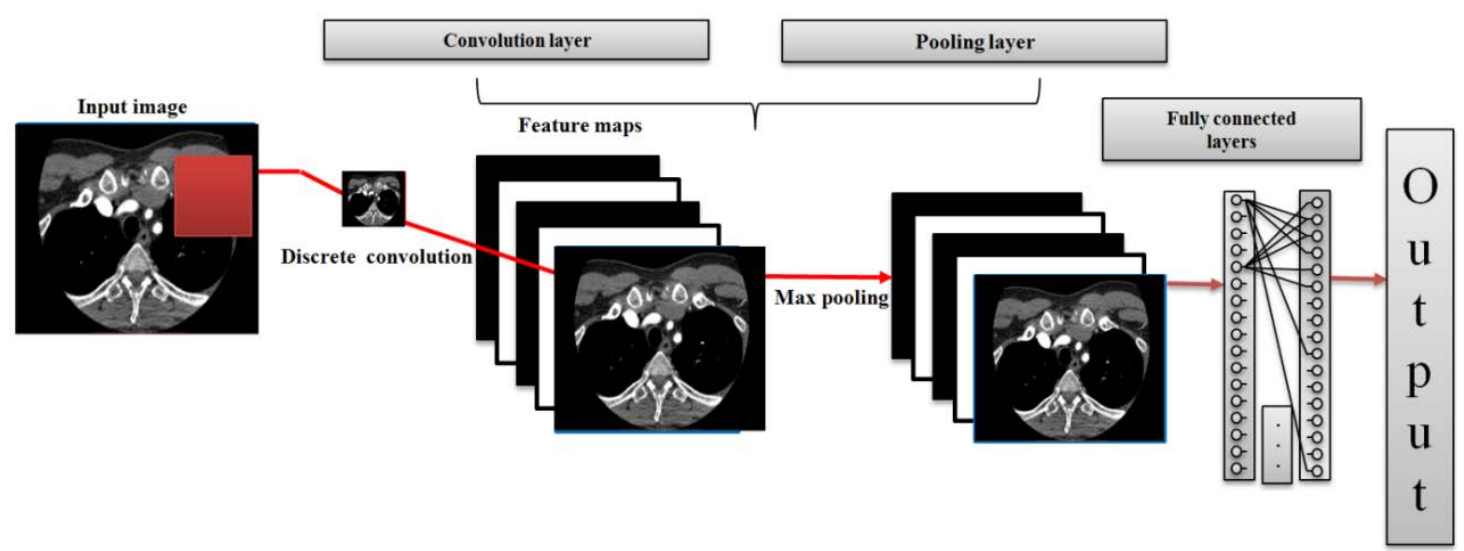

Figure 3. Diagram of Convolutional Network

\subsubsection{Convolutional Designs}

More recent work using convolutional neural networks (CNNs) allow to greatly reduce the number of model parameters compared to a fully connected network by applying convolutional operations to only small regions of the input space and by sharing parameters between regions (Figure 3) [34].

\section{Seven-year Vision}

It is likely that in a seven year time the level of automation for study and interpretation will be extensively raised and compared to what is feasible Nowadays. It is likely, that entirely unsupervised extraction of all image parameters will be possible for nuclear cardiology, and minimal supervision will be required for other modalities. Greater standardization of acquisition protocols will be needed to maximize the potential gains from automation and machine learning. This goal will require significant support from the vendors but also from the medical centres - to facilitate data sharing. Fully quantitative diagnostic and risk stratification scores will be developed for clinicians and these will become integrated with the imaging software. Risk stratification will transition from oversimplified population-based risk scores to machinelearning-based metrics incorporating a large number of clinical and imaging variables in real-time beyond the limits of human cognition - this will deliver highly accurate and individual personalized risk assessments and facilitate tailored management plans. However, the clinical translation of these exciting techniques will depend on many factors outside of technological progress, such as aspects related to logistical, legal, standardization, and reimbursement.

\section{Conclusion}

We hope to have given the readers an idea of why we need machine learning and what kind of data is available. The future of machine learning in cardiac electrophysiology, integrated with robotic catheter navigation systems, allowing for automated mapping and ablation, and perhaps eventually with real-time visualization of the lesions formed, to assist when complete lines of ablation lines have to be drawn.

\section{Acknowledgments}

We are thankful to Department of Biomedical Engineering, Chung Yuan Christian University, Taiwan and special thanks to Weichihhu lab members.

\section{Conflicts of Interest}

The authors have no relevant conflicts of interest to disclose.

\section{References}

[1] Thornton, Andrew. "Imaging in electrophysiology." SA Heart Journal 5.4 (2017): 166-170.

[2] Shinbane, Jerold S., et al. "CT imaging: cardiac electrophysiology applications." Cardiac CT imaging. Springer London, 2010. 293-308.

[3] Pullan, Andrew, David Paterson, and Fred Greensite. "Non-invasive imaging of cardiac electrophysiology." Philosophical Transactions: Mathematical, Physical and Engineering Sciences 2001: 1277-1286.

[4] Panutich, Michael S., and Bradley P. Knight. "Imaging techniques in cardiac electrophysiology." Expert review of cardiovascular therapy 4.1 (2006): 59-70.

[5] Bengio, Yoshua, Aaron Courville, and Pascal Vincent. "Representation learning: A review and new perspectives." IEEE transactions on pattern analysis and machine intelligence 35.8 2013: 1798-1828.

[6] LeCun, Yann, Yoshua Bengio, and Geoffrey Hinton. "Deep learning." Nature 521.7553 2015: 436-444.

[7] Schmidhuber, Jürgen. "Deep learning in neural networks: An overview." Neural networks 61 2015: 85-117.

[8] Rahman, Md Mahmudur, Prabir Bhattacharya, and Bipin C. Desai. "A framework for medical image retrieval using machine learning and statistical similarity matching techniques with relevance feedback." IEEE Transactions on Information Technology in Biomedicine 11.1 2007: 58-69.

[9] Kononenko, I. Machine learning for medical diagnosis: history, state of the art and perspective. Artificial Intelligence in medicine, 2001, 23(1), 89-109.

[10] Moher, D., Liberati, A., Tetzlaff, J., Altman, D. G., and Group, P., "Preferred Reporting Items for Systematic Reviews and MetaAnalyses: The PRISMA Statement,” Int. J. Surg., 2010, 8(5), pp. 336-341.

[11] Berchialla P, Foltran F, Bigi R, Gregori D. Integrating stressrelated ventricular functional and angiographic data in preventive cardiology: a unified approach implementing a Bayesian network. J Eval Clin Pract. 2012; 18: 637-643.

[12] Isgum I, Prokop M, Niemeijer M, Viergever MA, van Ginneken B. Automatic coronary calcium scoring in low-dose chest computed tomography. IEEE Trans Med Imaging. 2012; 31: 2322-2334. 
[13] Lee K, Zhu J, Shum J, Zhang Y, Muluk SC, Chandra A, Eskandari MK, Finol EA. Surface curvature as a classifier of abdominal aortic aneurysms: a comparative analysis. Ann Biomed Eng. 2013; 41: 562-576.

[14] Mohammadpour RA, Abedi SM, Bagheri S, Ghaemian A. Fuzzy rulebased classification system for assessing coronary artery disease. Comput Math Methods Med. 2015; 2015: 564867.

[15] Xiong G, Kola D, Heo R, Elmore K, Cho I, Min JK. Myocardial perfusion analysis in cardiac computed tomography angiographic images at rest. Med Image Anal. 2015; 24: 77-89.

[16] Knackstedt C, Bekkers SC, Schummers G, Schreckenberg M, Muraru D, Badano LP, Franke A, Bavishi C, Omar AM, Sengupta PP. Fully automated versus standard tracking of left ventricular ejection fraction and longitudinal strain: the FAST-EFs Multicenter Study. J Am Coll Cardiol. 2015; 66: 1456-1466.

[17] Arsanjani R, Dey D, Khachatryan T, Shalev A, Hayes SW, Fish M, Nakanishi R, Germano G, Berman DS, Slomka P. Prediction of revascularization after myocardial perfusion SPECT by machine learning in a large population. J Nucl Cardiol. 2015; 22: 877-884.

[18] Berikol GB, Yildiz O, Özcan IT. Diagnosis of acute coronary syndrome with a support vector machine. J Med Syst. 2016; 40: 84.

[19] Čelutkienė J, Burneikaitė G, Petkevičius L, Balkevičienė L, Laucevičius A. Combination of single quantitative parameters into multiparametric model for ischemia detection is not superior to visual assessment during dobutamine stress echocardiography. Cardiovasc Ultrasound. 2016; 14: 13.

[20] Motwani M, Dey D, Berman DS, Germano G, Achenbach S, AlMallah MH, Andreini D, Budoff MJ, Cademartiri F, Callister TQ, Chang HJ, Chinnaiyan K, Chow BJ, Cury RC, Delago A, Gomez M, Gransar H, Hadamitzky M, Hausleiter J, Hindoyan N, Feuchtner G, Kaufmann PA, Kim YJ, Leipsic J, Lin FY, Maffei E, Marques H, Pontone G, Raff G, Rubinshtein R, Shaw LJ, Stehli J, Villines TC, Dunning A, Min JK, Slomka PJ. Machine learning for prediction of all-cause mortality in patients with suspected coronary artery disease: a 5-year multicentre prospective registry analysis. Eur Heart J. 2017; 38: 500-507.

[21] Patel, Shailendra B., et al. "Three-dimensional rotational angiography imaging of double aortic arch vascular ring." Images in pediatric cardiology 2013.

[22] Sommer, Philipp, et al. "Non-fluoroscopic catheter tracking for fluoroscopy reduction in interventional electrophysiology." Journal of visualized experiments: JoVE 992015.
[23] Thornton, Andrew. "Imaging in electrophysiology." SA Heart Journal 5.4 ,2017: 166-170.

[24] Szili-Torok T, Kimman GJ, Scholten M, et al. Ablation lesions in Koch's triangle assessed by three-dimensional myocardial contrast echocardiography. Cardiovasc Ultrasound.2004 Dec 9; 2(1): 27.

[25] Jongbloed MR, Bax JJ, Lamb HJ, et al. Multislice computed tomography versus intracardiac echocardiography to evaluate the pulmonary veins before radiofrequency catheter ablation of atrial fibrillation: a head-to-head comparison. J Am Coll Cardiol. 2005 Feb 1; 45(3): 343-50.

[26] Packer DL. Three-dimensional mapping in interventional electrophysiology: techniques and technology. J Cardiovasc Electrophysiol. 2005 Oct; 16(10): 1110-6.

[27] Kistler PM, Earley MJ, Harris S, et al. Validation of threedimensional cardiac image integration: use of integrated CT image into electroanatomic mapping system to perform catheter ablation of atrial fibrillation. J Cardiovasc Electrophysiol. 2006 Apr; 17(4): 341-8.

[28] Dong J, Calkins H, Solomon SB, et al. Integrated electroanatomic mapping with three-dimensional computed tomographic images for real-time guided ablations. Circulation. 2006 Jan 17; 113(2): 186-94.

[29] Cireşan, D. C., Giusti, A., Gambardella, L. M., \& Schmidhuber, J. Mitosis detection in breast cancer histology images with deep neural networks. In International Conference on Medical Image Computing and Computer-assisted Intervention, 2013, (pp. 411418). Springer, Berlin, Heidelberg.

[30] McCulloch, W. S., \& Pitts, W. A logical calculus of the ideas immanent in nervous activity. The bulletin of mathematical biophysics, 1943, 5(4), 115-133.

[31] Farley, B. W. A. C., \& Clark, W. Simulation of self-organizing systems by digital computer. Transactions of the IRE Professional Group on Information Theory, 1954, 4(4), 76-84.

[32] Rosenblatt, F. The perceptron: A probabilistic model for information storage and organization in the brain. Psychological Review, 1958, 65(6), 386.

[33] LeCun, Y. Generalization and network design strategies. Connectionism in perspective, 1989, 143-155.

[34] Krizhevsky, A., Sutskever, I., \& Hinton, G. E. Imagenet classification with deep convolutional neural networks. In Advances in neural information processing systems, 2012, (pp. 1097-1105) 